\title{
O QUE É IMPORTANTE CUIDAR PARA MANTER A BOA SAÚDE? CONCEPÇÕES DE UNIVERSITÁRIOS DE UM CURSO SUPERIOR EM SAÚDE
}

\author{
WHAT IS IMPORTANT TO TAKE CARE TO MAINTAIN GOOD HEALTH? \\ CONCEPTIONS OF HEALTH STUDENTS FROM HIGHER EDUCATION
}

\author{
Maria Thereza Ávila Dantas Coelho*; Vanessa Prado Santos**; Maria Beatriz Barreto do \\ Carmo***; Natália Santana Reis Cerqueira****
}

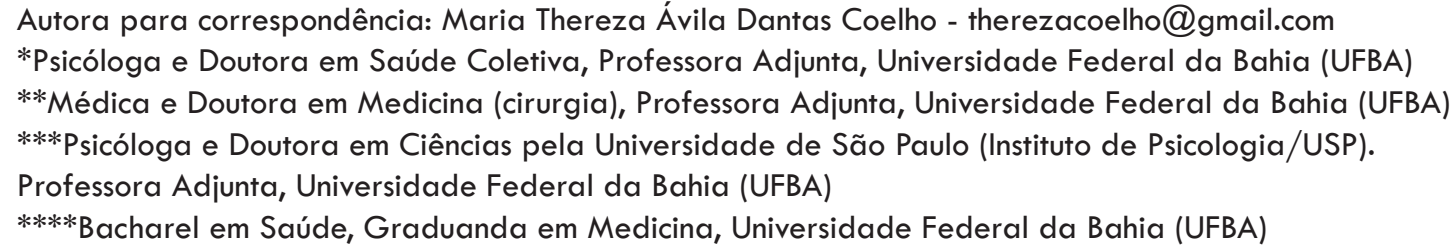

\section{R E S U M O}

\begin{abstract}
O cuidado à saúde pode ser entendido numa concepção mais tradicional, centrado em hábitos e comportamentos individuais, até uma concepção mais ampliada e afinada com a complexidade do processo saúde-doença, envolvendo uma noção mais avançada e social acerca da promoção da saúde. Visto que ambas as concepções apresentam consequências distintas para as práticas de atenção à saúde, faz-se importante conhecer a compreensão de estudantes da área acerca daquilo que consideram importante para a manutenção da saúde. Foi realizada uma revisão de literatura sobre o tema e uma análise qualitativa a partir das respostas à questão 'o que você considera importante cuidar para manter boa saúde?', que compõe o questionário de uma pesquisa maior, intitulada 'Concepções e práticas de saúde e doença', aplicado a 195 alunos de um curso superior de saúde. A pesquisa foi aprovada pelo Comitê de Ética em Pesquisa da Secretaria de Saúde da Bahia, através do fício $067 / 2010$. Todos os participantes declararam a sua anuência de participação e conhecimento dos objetivos da pesquisa, assinando o Termo de Consentimento Livre e Esclarecido (TCLE). Como resultados, a maioria dos discentes, $59 \%$, referiram a alimentação como prioritária. Quanto à atividade física, $48 \%$ responderam que esta é uma importante mantenedora da saúde. O cuidado com a saúde mental foi citado por $39 \%$ dos estudantes e a relevância dos aspectos socioculturais nesse processo, por $12 \% .16$ estudantes $(8,2 \%)$ apontaram consultas regulares e realização de exames periódicos como importantes para manter a saúde. As respostas apontam que os estudantes ingressam na Universidade com uma visão predominantemente individualista acerca dos fatores relacionados à saúde, apontando a necessidade de maior inserção do conteúdo social da promoção da saúde ampliada desde o ensino médio, além de maior divulgação na mídia e na educação superior.
\end{abstract}

Palavras-chave: Cuidado, saúde, educação superior. 
Health care can be understood in a more traditional way, centered on individual habits and behaviors, to a broader conception and attuned to the complexity of the health-disease process, involving a more advanced and social notion about health promotion. Since both concepts have distinct consequences for health care practices, it is important to know the understanding of health students about what they consider important for maintaining health. A literature review was conducted on the subject and a qualitative analysis from the answers to the question 'what you consider important to take care to maintain good health?', which makes up the questionnaire of a larger research entitled 'conceptions and practices of health and disease', applied to 195 health students from higher education. The research was approved by the Ethics Committee of the State Office of Health from Bahia with the document $067 / 2010$. All participants agreed with their participation and signed the free and informed consent. As a result, the majority of students, $59 \%$, reported feeding as a priority. As for physical activity, $48 \%$ responded that this is an important supporter of health. Mental health care was cited by $39 \%$ of students and the relevance of sociocultural aspects by $12 \% .16$ students $(8.2 \%)$ showed regular consultations and conducting periodic examinations as important to maintain health. The responses indicate that students enter the University with a predominantly individualistic view about the health-related factors, pointing to the need for greater integration of social content promoting expanded health since high school from higher education.

Keywords: Care, health, higher education. 


\section{INTRODUÇÃO}

O tema do cuidado coloca-se como central no campo da saúde, primeiro por desvelar um aspecto intrínseco à condição humana - a capacidade de cuidar -, e também por operacionalizar um conjunto de práticas, tecnologias e procedimentos voltados para a atenção à saúde'. Na primeira perspectiva, - cuidado é considerado como um aspecto fundamental da sobrevivência dos seres humanos, sendo indispensável aos indivíduos em particular, mas, sobretudo, à continuidade dos grupos sociais. Dito de outro modo, o cuidado constitui a própria existência humana e nos caracteriza e qualifica como seres eminentemente relacionais'.

Aprofundando essa perspectiva, temos a análise do filósofo Michel Foucault sobre uma noção complexa da Antiguidade Clássica, o cuidado de si, utilizada pelos Gregos para caracterizar um conjunto de atitudes e práticas concernentes ao fato de ocuparse e preocupar-se consigo mesmo ${ }^{2}$. 0 cuidado de si implicaria, portanto, uma responsabilização do sujeito consigo, pressupondo uma relação singular e ativa com a verdade e com o saber, bem como com a sua aplicação prática sobre a própria saúde 2 .

Na segunda perspectiva, como componente das práticas, o cuidado pode ser operacionalizado de diversas maneiras e a partir de arranjos distintos, com $\circ$ uso de tecnologias duras, leve-duras e leves, atuando nos níveis da promoção da saúde, prevenção de doenças, tratamento e recuperação ${ }^{3}$. Tanto $\circ$ uso de tecnologias quanto os níveis de operacionalização do cuidado dependerão da época e lugar, do contexto sociocultural e das necessidades em saúde colocadas em determinado tempo e espaço.

Atualmente, o tema do cuidado vem sendo abordado a partir de uma concepção ampliada, em sintonia com o conceito ampliado de saúde e do próprio processo saúde-doença ${ }^{4,5}$. $O$ conceito ampliado de saúde abre, por sua vez, um importante espaço para o aprofundamento da noção de promoção da saúde, conferindo-lhe destaque em relação aos demais níveis de cuidado mais amplamente reconhecidos e difundidos, inclusive no senso comum, quais sejam, a prevenção de doenças e as estratégias de cura/ reabilitação.
Em linhas gerais, podemos afirmar que a promoção da saúde parte de um olhar positivo sobre a saúde, ou seja, enfatiza os aspectos necessários para mantê-la, potencializá-la e promovê-la. A diversidade das concepções sobre esse termo possibilita agrupá-las sob dois aspectos centrais distintos ${ }^{6}$. No primeiro, a promoção da saúde tem como foco os comportamentos, hábitos e estilos de vida dos indivíduos. Assim, suas ações tendem a ser eminentemente educativas, associadas ao comportamento individual, como a dieta e atividade física, por exemplo. De acordo com Buss ${ }^{6}$, nessa abordagem, tudo aquilo que está fora do alcance dos indivíduos não compõe o cenário da promoção da saúde. No segundo grupo, alinhado a uma concepção mais social da promoção da saúde, - foco está nos determinantes gerais do processo saúde-doença, aqueles sobre os quais o indivíduo não tem controle direto, mas dentro dos quais a sua vida transcorre ${ }^{7}$. As transformações e ampliação do termo acompanham, também, as mudanças ocorridas na própria definição e entendimento sobre a saúde.

Desde a Conferência de Ottawa (realizada em Ottawa, Canadá, em 1986) ${ }^{8}$, marco das discussões sobre o tema, a promoção da saúde vem sofrendo alterações em sua definição e no modo como é operacionalizada, destacando-se a necessidade das políticas públicas saudáveis e Ações do Estado, da comunidade, de indivíduos, do sistema de saúde e de parcerias intersetoriais. Dessa forma, ela interfere direta e indiretamente em um conjunto de necessidades da população, como alimentação, educação, habitação, recursos sustentáveis, renda, justiça social, entre outros determinantes sociais ${ }^{7}$.

Posto este panorama e para além das diferentes abordagens, a promoção da saúde coloca-se numa direção oposta ao que prega a concepção biomédica do processo saúde-doença, caracterizada pelo reducionismo da determinação dos problemas de saúde aos fatores biológicos e alheios aos indivíduos ${ }^{4}$. Nesta concepção o foco está na patologia e nas estratégias de cura, enquanto que na promoção da saúde o foco é justamente a saúde e as estratégias para produzi-la no tecido social e promovê-la no nível individual. Nesse sentido, ela ultrapassa os limites do biológico, enxergando tanto a individualidade de cada ser, como a totalidade do ambiente em que o sujeito se insere, levando em 
consideração as dimensões econômicas, sociais e políticas ${ }^{6}$.

Em consonância com a ideia anteriormente exposta, a formação dos profissionais de saúde passou a ser repensada diante da necessidade de que estes estejam capacitados para promover a saúde, e não apenas para prevenir e tratar doenças. Diante da necessidade de serem implementadas formações na área mais alinhadas à complexidade do objeto saúde, bem como promotoras de práticas que extrapolem o referencial curativista, desenvolve-se - projeto do Bacharelado Interdisciplinar em Saúde (BIS), implantado em 2009 pela Universidade Federal da Bahia (UFBA), baseado em uma proposta de reforma universitária denominada "Universidade Nova"?.

O projeto pedagógico do Bacharelado Interdisciplinar (BI) se baseia no regime de ciclos, segundo o qual o primeiro ciclo é constituído pelos Bacharelados Interdisciplinares de Saúde, Ciência e Tecnologia, Humanidades e Artes; o segundo ciclo, pelos cursos profissionalizantes; e o terceiro, por mestrados e doutorados. Essa trajetória diferencia o estudante que pretende seguir a carreira acadêmica ou ingressar no mercado de trabalho, visto que terá uma formação ampla e interdisciplinar ${ }^{10}$.

Essa transformação aponta para a relevância das diferenças culturais atuais e para a maior flexibilidade da educação superior ${ }^{11,12}$.

Nessa direção, ○ Bacharelado Interdisciplinar em Saúde visa responder e/ou problematizar os saberes e as práticas de saúde da contemporaneidade, que hoje são exercidas por profissionais com uma formação especializada e disciplinar. A partir dessa perspectiva, é possibilitada, aos seus egressos, uma compreensão abrangente da problemática de saúde nas sociedades contemporâneas e o desenvolvimento de competências e habilidades cognitivas, referentes à ação no campo da saúde, e valorativas, que incluem compromissos éticos ${ }^{13}$.

Diante da importância da compreensão ampliada das práticas de saúde em nossa sociedade, em especial no âmbito da formação na área da saúde, este artigo objetivou identificar e analisar o que os alunos do BIS, que ingressaram em 2013, consideram importante para a manutenção da saúde.
MÉTODO

Neste estudo utilizou-se a abordagem metodológica qualitativa, pela finalidade de acessar de forma aprofundada as opiniões e representações sobre uma temática em questão ${ }^{14}$. Como instrumento de coleta de dados, foi utilizado um questionário composto por onze perguntas em torno de temas relacionados a concepções e práticas de saúde e doença. Participaram desta pesquisa 195 estudantes recém ingressos no curso em 2013. Visando conhecer $\circ$ que os estudantes entendem como elementos e aspectos mantenedores da saúde, foram selecionadas as respostas dadas à seguinte pergunta: 'o que você considera importante cuidar para manter boa saúde?'. A escolha dessa questão se deu pelo fato de a mesma emular a noção de promoção de saúde, ao trazer as noções de cuidado e manutenção, associadas a uma perspectiva positiva da saúde, com abertura, inclusive, para a consideração dos aspectos macro-sociais.

Com a finalidade de responder ao problema apresentado, utilizou-se a análise de conteúdo temática para a análise dos dados. Nesse sentido, seguiram-se as seguintes etapas sugeridas por Minayo ${ }^{15}$ : Pré-Análise; Leitura Flutuante; Constituição do Corpus; Exploração do Material e Tratamento dos resultados obtidos e interpretação.

A partir da exploração dos resultados, foram criadas três categorias analíticas, a saber: 'Alimentação e Atividade Física', 'Realização de Consultas e Exames Periódicos' e 'Cuidado Bio-Psico-Social'. Em seguida, discutiu-se os significados e inter-relações entre as respostas dos estudantes e o cuidado à saúde, na atualidade.

Este estudo foi aprovado pelo Comitê de Ética em Pesquisa da Secretaria de Saúde da Bahia, através do ofício $067 / 2010$. Todos os participantes declararam a sua anuência e conhecimento dos objetivos da pesquisa, assinando o Termo de Consentimento Livre e Esclarecido (TCLE). 


\section{RESULTADOS}

\section{Alimentação e Atividade Física}

Esta categoria se propõe a analisar a representação dos estudantes sobre a atividade física e a alimentação como mantenedoras da saúde. A relação mais evidente acerca disso pode ser visualizada através da maior frequência de respostas correlacionando a manutenção da saúde com a alimentação (115 estudantes, 59\%) e a atividade física (93 estudantes, $48 \%$ ).

Alimentar-se bem, fazer as três refeições diárias respeitando seu horário, fazer atividades físicas, beber bastante água.

(Informante 115)

Boa alimentação, prática regular de exercício, atenção com o seu corpo e seu funcionamento. (Informante 16)

Boa alimentação e práticas de atividade física. (Informante 24)

Outro aspecto evidenciado nesta categoria referese ao enfoque no 'corpo físico' ou na 'forma física' como importante para a manutenção da saúde:

Cuidado com alimentação, higiene, forma física. (Informante

A forma física e o coração. (Informante 66)

\section{Realização de Consultas e Exames Periódicos}

Esta categoria refere-se a um conjunto de ações e comportamentos identificados nas respostas de 16 estudantes $(8,2 \%)$ como importantes à manutenção da saúde, e que envolvem consultas regulares a médicos e realização de exames periódicos, visando diagnósticos precoces, como explicitado nos trechos abaixo:

Primeiramente, estar regularmente se consultando aos médicos para que ele possa diagnosticar alguma doença suspeita. (Informante 26)

Consultar sempre um médico (...). (Informante 63)

Realizar exames e consultas periódicas (....). (Informante 17)

Exames regulares (...). (Informante 29)

\section{Cuidado Bio-Psico-Social}

Esta categoria se propõe a analisar as respostas dos discentes relacionadas à importância de outros fatores, para além da alimentação, atividade física e realização de consultas e exames, relacionados a duas ou mais dimensões da tríade biopsicossocial e à manutenção e cuidado da saúde. Na presente categoria, o cuidado com a saúde mental apareceu nas respostas de 75 discentes (39\%) como sendo importante para manter a saúde:

O corpo (através de uma boa alimentação e exercícios físicos) e a mente (estando bem consigo e com o meio que se vive). (Informante 22)

Alimentação / corpo - físico e no mental. (Informante 178)

Além dos aspectos de ordem psicológica/subjetiva, surgiram também referências aos fatores sociais, no entanto de forma bastante tímida entre os estudantes. Chamou atenção o fato de apenas 24 discentes $(12 \%)$ terem referido o caráter social como fator importante para a manutenção da saúde, como descrito a seguir:

Acredito que para manter uma boa saúde é preciso cuidar-se de maneira integral, garantindo bem-estar biopsicossocial. (Informante 48)

A junção entre cuidar do físico, psíquico e social. (Informante

Alimentação, rotina, convívio social. (Informante 33)

Psicológico e Social. (Informante 175)

\section{DISCUSSÃO}

No que se refere à categoria 'Alimentação e Nutrição', é importante notar que as falas dos estudantes são reveladoras de padrões normativos relacionados aos hábitos e rotinas alimentares e de atividade física, conforme explicitado nas respostas, a exemplo de: "Alimentar-se bem, fazer as três refeições diárias respeitando seu horário, fazer atividades físicas, beber bastante água" (Informante 115). Apesar da grande quantidade de informação direcionada à população pela mídia, com normas e padrões sobre alimentação e atividade física, observa-se crescentes índices de obesidade ${ }^{16}$, por 
exemplo, sendo que tal fato revela que apenas a informação não é suficiente para a mudança de determinados padrões, devendo-se considerar, especialmente no contexto brasileiro, a importância dos Determinantes Sociais da Saúde ${ }^{7}$, que modelam as possibilidades da população de reproduzir ou não esses padrões ditos saudáveis. Segundo Carvalho, Pinheiro e Recine ${ }^{17}$, a prática de atividade física, como meio de promover saúde, não deve culpabilizar o sedentarismo, nem se impor sem levar em consideração os condicionantes socioeconômicos. Portanto, é nesse contexto de um país com grandes desigualdades socioeconômicas e culturais que a manutenção da boa saúde pela população deve considerar, também, a sua determinação social, e não apenas imprimir no nível individual o seu protagonismo e responsabilização.

É importante notar também que as referências feitas à alimentação e atividade física como relacionadas à manutenção da saúde não agregaram valor cultural a essas práticas. Pressupondo que a maneira pela qual se constroem os valores culturais são diferentes, e que o fator sociocultural repercute nas representações tanto alimentares quanto corporais $^{18}$, não podemos definir ou inferir $\circ$ significado de "boa alimentação" nem de "prática regular de exercícios" na percepção dos discentes, já que os mesmos não explicitaram seus critérios de valoração dessas práticas. Foi observado que os estudantes não definiram o que consideravam como alimentação saudável, qualificando-a apenas como "boa", "adequada" e com outros adjetivos correspondentes, o mesmo ocorrendo em relação à atividade física, reproduzindo assim um padrão normativo de saúde difundido na sociedade e apreendido pelo senso comum. Ainda sobre esse aspecto, chamou a atenção o fato de o esporte ter sido pouco relacionado à prática de exercícios e à atividade física.

Outro aspecto a ser destacado diz respeito à referência feita amiúde ao 'corpo físico' ou 'forma física'. Em nossos dias, a representação do corpo é atravessada por sua excessiva exposição e também pela valorização do belo, tendo sua expressão máxima na denominada 'ditadura da magreza' ou "do corpo perfeito"19. Essa nova maneira de lidar com o corpo está fundamentada em dois pilares, que são a alimentação e a atividade física, práticas frequentemente padronizadas, normatizadas, controladas e estimuladas pela mídia, ultrapassando assim diversas barreiras culturais e sociais ${ }^{19}$. Portanto, quando os estudantes referem-se à 'boa alimentação' ou 'prática regular' de atividade física, entendemos que as mesmas refletem os padrões normatizados e profundamente difundidos na atualidade.

Em relação à categoria Realização de consultas e exames periódicos, num contexto marcado pela hegemonia do modelo biomédico, eminentemente curativista e dominado pelas tecnologias duras ${ }^{3}$, - check-up (exames periódicos) e screening (rastreamento de doenças) ocupam lugar de destaque dentre as estratégias de cuidado $^{20}$. Essa prática encontra sua sustentação na ideia amplamente difundida de que o avanço tecnológico é capaz de identificar precocemente as doenças e aumentar significativamente as chances de um tratamento bem sucedido, ainda que, conforme Martins $^{21}$, poucos são os exames comprovadamente eficazes como estratégia de rastreamento.

As respostas dos estudantes apontam, portanto, os exames e consultas como estratégias de prevenção e, por conseguinte, de manutenção da saúde. Fazendo um breve resgate conceitual, identificamos o termo prevenir com o sentido de evitar, de se antecipar ao dano, evitando o seu progresso e impedindo que se realize ${ }^{20}$. Segundo Czeresnia ${ }^{20}$, "as ações preventivas definem-se como intervenções orientadas a evitar $\circ$ surgimento de doenças específicas, reduzindo sua incidência e prevalência nas populações".

Diagnóstico e tratamento precoce, por sua vez, estão situados dentro do reconhecido modelo de Leavell e Clark ${ }^{22}$, na fase da prevenção secundária. Neste modelo, que compreende três fases, a prevenção primária é representada pelas estratégias de promoção e proteção da saúde, anteriores à ocorrência da doença; a prevenção secundária (representando a prevenção propriamente dita) é composta pelo diagnóstico e tratamento precoce, anterior ao sintoma, e a prevenção terciária se refere às ações de cura e reabilitação.

A centralidade da prevenção secundária, observada nas respostas dos estudantes, indica uma fragilidade no entendimento da promoção da saúde como uma estratégia de, em última 
instância, produção e manutenção da saúde. Tal fragilidade acompanha a sua pouca valorização no senso comum, embora tenhamos uma política pública específica para a Promoção da Saúde ${ }^{23}$. Tal resultado indica a necessidade dessa discussão ser realizada nos cursos de formação superior em saúde, priorizando assim o entendimento do modelo amplo de ações promotoras de saúde e a compreensão crítica acerca da vinculação entre as estratégias de prevenção secundária (baseadas nas tecnologias médicas de ponta) e a mercantilização do setor.

Na categoria Cuidado Bio-Psico-Social, embora os aspectos de ordem emocional/mental/psicológica e subjetiva tenham sido observados, as respostas dos estudantes evidenciaram uma concepção dicotômica, representando o corpo e a subjetividade como independentes. A idealização de ambos como 'fenômenos' distintos subjaz à prática médica moderna. Embora possamos observar sinais de mudança na visão médica quanto a essa separação entre mente e corpo, este é ainda o referencial epistemológico paradigmático a partir do qual as concepções de sujeito, de saúde e doença, bem como as práticas de cuidado estão fundamentadas na contemporaneidade ${ }^{24}$.

No que se refere aos aspectos eminentemente sociais, a sua consideração desmistifica as práticas de saúde como uma realidade individual e biológica. Especialmente num contexto de profunda desigualdade de condições de vida, como o brasileiro, a consideração dos Determinantes Sociais em Saúde revela a importância dos elementos dentro dos quais a vida transcorre, sendo estes considerados 'as causas das causas' dos processos de adoecimento, a exemplo do local de moradia, renda, tipo de emprego, saneamento, escolaridade, etc $^{7}$. No entanto, as referências ao social observadas nas respostas dos estudantes, além de escassas, não remetem a esse nível de análise, apresentando-se, por sua vez, de forma genérica, acompanhando a tríade biopsicossocial, já tão difundida no senso comum como sendo a forma mais adequada de se referir à saúde, mas sem que haja reflexão ou entendimento claro a esse respeito.

\section{CONCLUSÃO}

Observou-se, nesta pesquisa, que as concepções dos estudantes sobre os aspectos que consideram necessário cuidar para manter a saúde, ou seja, promovê-la, em última instância são permeadas por elementos relacionados ao corpo e ao nível individual de ação (alimentação, atividade física e realização de exames). Adicionalmente, cabe destacar que foi predominante a concepção de uma relação dicotômica e estanque entre corpo e mente, não estando ambos integrados e correlacionados para a produção do bem-estar geral. Ficou evidente ainda a fragilidade da referência aos fatores socioeconômicos e culturais, o que revela uma dissociação entre as dimensões individual e social geral, apontando para um não reconhecimento claro dos aspectos de nível macro, sejam eles sociais, econômicos ou culturais, na manutenção e promoção da saúde.

A partir desta análise, conclui-se que os estudantes, ao ingressarem no curso, pensam a saúde de maneira individualizada, centrada no espaço corporal e dependente de fatores comportamentais e autônomos, em descompasso com o meio social. Essa noção corrobora, em grande medida, a perspectiva difundida no senso comum, por sua vez baseada no modelo biomédico hegemônico. Portanto, esse é um aspecto que se coloca como central para ser trabalhado ao longo da formação superior, estando alinhado à proposta do BIS de refletir questões relacionadas à saúde de forma complexa, ampliada e interdisciplinar, conforme a direção das políticas de saúde de nosso país. Por fim, a partir deste estudo, apontamos a importância de se investigar as concepções de cuidado e manutenção da saúde, bem como de promoção da saúde ampliada, entre discentes de outros cursos superiores em saúde, para melhor direcionar a sua formação profissional e refletir acerca da difusão de nossa Política Nacional de Promoção da Saúde no contexto brasileiro. 


\section{REFERÊNCIAS}

1. Ayres JRCM. O cuidado, os modos de ser (do) humano e as práticas de saúde. Saúde e Sociedade. 2004;13(3):16-29. doi: 10.1590/S010412902004000300003

2. Foucault M. Hermenêutica do sujeito. São Paulo: Martins Fontes; 2004

3. Merhy EE, Feverwerker LCM. Novo olhar sobre as tecnologias de saúde: uma necessidade contemporânea. In: Mandarino ACS, Gomberg E. (Orgs.). Leituras de novas tecnologias e saúde. São Cristóvão: Editora UFS; 2009. P.29-74

4. Batistella C. Abordagens contemporâneas do conceito de saúde. In: Fonseca AF (org.). O território e o processo saúde-doença. Rio de Janeiro: EPSJV, Fiocruz; 2007. P.51-86

5. Scliar M. História do conceito de saúde. Rev. Saúde Coletiva. 2007;17(1):29-42

6. Buss PM. Promoção da Saúde e Qualidade de Vida. Ciência \& Saúde Coletiva. 2000;5(1): 163-177. doi: $10.1590 / \mathrm{S} 1413-81232000000100014$

7. Buss PM, Filho AP. A saúde e seus determinantes sociais. Rev. Saúde Coletiva. 2007;17(1): 77-93. doi: $10.1590 /$ S0103-73312007000100006

8. World Health Organization. The Ottawa charter for health promotion. Geneve: WHO; 1986

9. Almeida-Filho N. Universidade nova: textos críticos e esperançosos. $1^{a} \mathrm{Ed}$. Brasília: Editora da Universidade de Brasília; 2007

10. Teixeira CF, Coelho MTAD. A construção do Projeto Político-Pedagógico do BI em Saúde: Transformando um sonho em realidade. In: Coelho MTAD, Teixeira CF. Uma experiência inovadora no ensino superior: Bacharelado Interdisciplinar em Saúde. Salvador: EDUFBA; 2014. P.53-72

11. Santos BS, Almeida Filho N. A Universidade Nova no Século XXI: Para uma Universidade Nova. Coimbra: Almedina; 2008

12. Teixeira CFS, Coelho MTAD, Rocha MND.

Bacharelado Interdisciplinar: Uma proposta Inovadora na educação superior em saúde no Brasil. Ciência \& Saúde Coletiva. 2013;18(6):1635-46. doi: 10.1590/

\section{S1413-81232013000600015}

13. Universidade Federal da Bahia. (UFBA). IHAC. Projeto Pedagógico do Bacharelado Interdisciplinar em Saúde. Salvador: UFBA; 2010

14. Bardin L. Análise de conteúdo. Lisboa: Edições 70; 1979

15. Minayo MCS. O desafio do conhecimento: pesquisa qualitativa em saúde. 12. ed. São Paulo: Hucitec; 2010

16. Malta DC, Andrade SC, Claro RM, Bernal RTI, Monteiro CA. Evolução anual da prevalência de excesso de peso e obesidade em adultos nas capitais dos 26 estados brasileiros e no Distrito Federal entre 2006 e 201 2. Rev Bras Epidemiol. 2014;17(1):267-276. doi: $10.1590 / 1809-4503201400050021$

17. Carvalho MFCC, Pinheiro ARO, Recine EG. As práticas alimentares saudáveis: $A$ promoção da saúde no contexto da segurança alimentar e nutricional. In: Castro A, Malo M. SUS: ressignificando a promoção da saúde. São Paulo: HUCITEC; 2006. P.87-207

18. Maciel ME. Olhares antropológicos sobre a alimentação: Identidade cultural e alimentação. Rio de Janeiro: Editora Fiocruz; 2005

19. Santos LAS. O corpo, o comer e a comida: um estudo sobre as práticas corporais alimentares cotidianas a partir da cidade de Salvador-Bahia. Salvador: EDUFBA; 2008

20. Czeresnia D. O Conceito de Saúde e a Diferença entre Prevenção e Promoção. In: Czeresnia D, Freitas $C M$ (Org.). Promoção da saúde: conceitos, reflexões, tendências. Rio de Janeiro: Editora Fiocruz; 2003. P. $39-53$

21. Martins MA. Check-up do check-up. Rev. Assoc. Med. Bras. 2005;51(3):121. doi: 10.1590/S010442302005000300001

22. Leavell H, Clark GG. Medicina preventiva. Rio de Janeiro: Mcgraw-Hill Ltda do Brasil; 1978

23. Brasil. Ministério da Saúde. Política Nacional de Promoção da Saúde. $3^{a}$ Ed. Brasília: Ministério da Saúde; 2010

24. Luz MT. Natural, racional, social: razão médica e racionalidade científica moderna. Rio de Janeiro: Campus; 1988 